\title{
A standardized set of 260 pictures for Modern Greek: Norms for name agreement, age of acquisition, and visual complexity
}

\author{
María Dimitropoulou and Jon Andoni Duñabeitia \\ Universidad de La Laguna, La Laguna, Spain \\ Panagiotis Blitsas \\ National and Kapodistrian University of Athens, Athens, Greece \\ AND \\ Manuel Carreiras \\ Basque Center on Cognition, Brain and Language, Donostia-San Sebastián, Spain \\ and Universidad de La Laguna, La Laguna, Spain
}

\begin{abstract}
The appropriate selection of both pictorial and linguistic experimental stimuli requires a previous languagespecific standardization process of the materials across different variables. Considering that such normative data have not yet been collected for Modern Greek, in this study normative data for the color version of the Snodgrass and Vanderwart picture set (Rossion \& Pourtois, 2004) were collected from 330 native Greek adults. Participants named the pictures (providing name agreement ratings) and rated them for visual complexity and age of acquisition. The obtained measures represent a useful tool for further research on Greek language processing and constitute the first picture normative study for this language. The picture norms from this study and previous ones may be downloaded from brm.psychonomic-journals.org/content/supplemental.
\end{abstract}

A thorough standardization process is fundamental to ensuring the quality of the experimental materials used in cognitive psychological research. To guarantee that empirical findings are valid and generalizable, the pictorial and linguistic materials used have to be previously standardized in a language-specific manner (see Sanfeliu \& Fernandez, 1996; Yoon et al., 2004). That is, to effectively control for differences across materials, a large pool of native speakers has to provide normative data for those materials first. In spite of the importance of languagespecific norms for pictures that are commonly used in psychological research, no such norms are available for Modern Greek. In the present study, the colorized version of the Snodgrass and Vanderwart (1980) picture data bank (S\&V, hereafter), created by Rossion and Pourtois (2004), was viewed by Greek-speaking young adults who provided norms concerning name agreement, age of acquisition (AoA), and visual complexity. The following sections include a brief overview of the relevant literature as well as a description of the variables measured in the present study.

Cross-cultural standardization of a set of materials that is widely used in psycholinguistic research has its exponent in the S\&V set of pictures. Snodgrass and Vanderwart (1980) created a set of 260 black-and-white line drawings that were initially standardized by AmericanEnglish-speaking participants. By now, this same set of pictures has been normalized across different languages (e.g., British English-Barry, Morrison, \& Ellis, 1997; Spanish-Cuetos, Ellis, \& Alvarez, 1999; Sanfeliu \& Fernandez, 1996; French-Alario \& Ferrand, 1999; Japanese-Nishimoto, Miyawaki, Ueda, Une, \& Takahashi, 2005; Icelandic-Pind, Jónsdóttir, Tryggvadóttir, \& Jónsson, 2000; Italian-Nisi, Longoni, \& Snodgrass, 2000; Dutch-Martein, 1995) and across different populations (e.g., children of different age groups; Berman, Friedman, Hamberger, \& Snodgrass, 1989; Cycowicz, Friedman, Rothstein, \& Snodgrass, 1997). Pictures from the S\&V set have been widely used in different areas of cognitive psychological research, such as object perception (e.g., Dell'Acqua, Job, \& Grainger, 2001), attention (e.g., Pashler \& Harris, 2001), and memory (e.g., Suzuki et al., 2002), and have become a recurrent tool, especially in psycholinguistic research. Psycholinguists have used these pictures to study phonological, lexical, and semantic processes in language perception and production (e.g., Belke, Brysbaert, Meyer, \& Ghyselinck, 2005; Damian \& Martin, 1998; Schriefers, Meyer, \& Levelt, 1990) in both monolinguals and bilinguals (see Hoshino \& Kroll, 2008; Knupsky \& Amrhein, 2007). Furthermore, the S\&V

M. Dimitropoulou, mdimitro@ull.es 
picture data bank has been extensively used in a variety of experimental paradigms, such as picture naming, pictureword interference tasks (e.g., Damian \& Martin, 1998; Schriefers, Jescheniak, \& Hantsch, 2002), and the visualworld paradigm (e.g., Duñabeitia, Avilés, Afonso, Scheepers, \& Carreiras, 2009; Huettig \& Altmann, 2005).

Despite their extended use, the black-and-white line drawings of the $\mathrm{S} \& \mathrm{~V}$ database provide a limited amount of visual information as compared with objects in real life and do not offer information about essential visual characteristics of the objects, such as color or texture ${ }^{1}$ (see Price \& Humphreys, 1989). Rossion and Pourtois (2004) compared name agreement values and naming latencies across groups of French adults who viewed either the line drawings of the $S \& V$ picture set or the same pictures graphically manipulated to include detailed texture and color information. These authors found that the addition of color and texture information provided a clear advantage for both the name agreement rates (i.e., more homogeneous responses) and the picture naming latencies (i.e., faster recognition and naming latencies) as compared with the original line drawings (see also Weekes, Shu, Hao, Liu, \& Tan, 2007). For this reason, in the present study the colorized and texturized version of the $\mathrm{S} \& \mathrm{~V}$ set provided by Rossion and Pourtois was used for standardization purposes.

The variables measured in the present study were name agreement, visual complexity, and AoA. These variables (among others) represent a constant in studies providing norms for the S\&V database (see Alario et al., 2004, for a review). In fact, as shown by Alario and colleagues, these three variables, together with image agreement and word frequency, were found to be the most effective predictors of picture naming latencies. ${ }^{2}$ Next, a short description of the measured variables is provided.

Name agreement refers to the degree to which participants agree on the name of a picture. The most commonly used name agreement measures are the percentage of participants giving a name for a particular picture, on the one hand, and the information statistic $H$ (Shannon, 1949), which reflects the level of agreement or discrepancy across participants for the given answers, on the other hand. As previously mentioned, Alario et al. (2004) found name agreement to be one of the strongest predictors of picture naming latencies (see also Barry et al., 1997; Bonin, Chalard, Méot, \& Fayol, 2002; Ellis \& Morrison, 1998; Nishimoto et al., 2005; Snodgrass \& Yuditsky, 1996; Vitkovitch \& Tyrell, 1995).

Visual complexity corresponds to the subjective evaluation of the number of lines and details in a drawing. A number of studies have reported longer naming latencies for pictures with higher visual complexity values (e.g., Alario et al., 2004; Ellis \& Morrison, 1998; Lloyd-Jones \& Nettlemill, 2007), highlighting the impact of this variable on picture naming. Visual complexity is proposed to affect naming latencies especially when the pictorial material is colored, as is the case in the present study (see Biederman, 1987; Paivio, Clark, Digdon, \& Bons, 1989). Thus, this variable should be controlled for in tasks involving picture presentation, and especially when more than one picture is simultaneously displayed. For instance, in the visual-world paradigm (see Altmann \& Kamide, 2007, for a review), participants are presented with several pictures on a screen, while their eye movements toward these images are being recorded. Considering that visual attention is highly driven by the saliency of the displayed pictures, visual complexity is a factor that has to be unavoidably controlled for in this paradigm (e.g., Huettig \& Altmann, 2005).

Age of acquisition (AoA) of a given item has also been proved to influence performance in word production and visual word recognition tasks (e.g., Barry et al., 1997; Dent, Johnston, \& Humphreys, 2008; Lachman, 1973; Lachman, Shaffer, \& Hennrikus, 1974; Morrison \& Ellis, 2000; Snodgrass \& Yuditsky, 1996; for similar evidence on Greek, see Bogka et al., 2003). AoA is positively correlated with reaction times in picture naming, reading aloud, and lexical decision tasks, suggesting that concepts that have been acquired earlier (namely, concepts with low AoA values) are processed faster than those acquired later in life (concepts with high AoA values). In that sense, a recent study by Cortese and Khanna (2007) showed that AoA is the most important predictor of word naming and lexical decision performance, over and above 22 other factors.

\section{The Present Normative Study}

The present study aimed at providing researchers with full picture naming norms for Modern Greek. Greek is the official language in Greece and Cyprus and is fluently spoken by more than 15 million individuals around the world. The specific orthographic and phonological properties of Greek make it especially relevant for psycholinguistic research (e.g., transparent orthography, unique script). In spite of this, Greek is one of the most understudied European languages. Efforts to provide empirical data on Greek language processing have been recently initiated (e.g., Ktori \& Pitchford, 2008; Orfanidou \& Sumner, 2005; Protopapas, Archonti, \& Skaloumbakas, 2007). However, to efficiently control for the materials, normative databases on Modern Greek are required, both for printed words and for pictures. GreekLex (Ktori, van Heuven, \& Pitchford, 2008), a recently created lexical database providing a series of lexical indexes on Greek (e.g., word frequency, length, number of orthographic neighbors), represents a step forward for the standardization of useful psycholinguistic statistics for Greek. The present study aims at providing Greek norms regarding name agreement, AoA, and visual complexity for the 260 pictures of the colorized and texturized Rossion and Pourtois (2004) picture set.

\section{METHOD}

\section{Participants}

Three hundred thirty undergraduates from the Informatics Department of the National and Kapodistrian University of Athens participated in the study. All were native speakers of Greek and participated in the study in exchange for course credit.

\section{Materials}

The Rossion and Pourtois (2004) colorized version of the S\&V 260-object data set was used for the collection of both the picture naming and the free-association norms. This set of pictures was created by Rossion and Pourtois by scanning and "cleaning" the origi- 
nal S\&V black-and-white line drawings, and then adding surface and color information using Adobe Photoshop 5.0. The whole set of pictures was acquired from the authors' Web site (www.cog.brown .edu/_tarr/stimuli.html).

\section{Procedure}

Instructions were given to the whole group of participants both orally and via a written form, along with the answer sheet. The instructions closely followed those adopted by Alario and Ferrand (1999) and Bonin, Peereman, Malardier, Méot, and Chalard (2003) in parallel studies. In the name agreement task, participants were instructed to identify each picture and to write down its name as it first came to their mind. In case they did not know the object or its name, participants were asked to give $D K$ (corresponding to don't know) as a response. In the AoA rating task, participants had to give an estimate of the age at which they thought they had learned each of the names in its written or oral form. They were provided with a scale with values from 1 to 5 corresponding to 3 -year age bands. 1 corresponded to a word learned between 0 and 3 years and 5 to one learned at 12 years or later. In the visual complexity rating task, participants were instructed to evaluate the visual complexity of each drawing on a scale from 1 (very simple) to 5 (very complex). They were explicitly told to rate the visual complexity of the object not in real life but in the drawing. All the participants completed the study at their own pace during nonteaching hours, by filling in a Microsoft Excel document accessible to them via the virtual e-class (electronic classroom) platform. Along with this file, an attached document containing the full set of instructions was always provided. The Microsoft Excel file contained the whole set of pictures, together with empty slots for collecting the written responses. The entire Microsoft Excel file containing the pictures was protected against modification, except for the empty response boxes that had to be filled by the participants. An "OK" sign appeared after participants provided each response, so that they knew that they could move to the subsequent response box. Upon completion of the questionnaire, the Microsoft Excel documents stayed saved at the virtual e-class platform, accessible only to the researchers. Participants were instructed to work as fast as possible, responding to the stimulus series in order of appearance. It was made explicit that there were no correct or incorrect answers. Two examples in which filler stimuli and responses were used were presented to further clarify the tasks. The order of appearance of the 260 pictures in the study was randomized to avoid semantic category sequence effects (to this end, four different lists were created).

\section{RESULTS AND DISCUSSION}

Each participant was asked to give 780 responses (260 pictures $\times 3$ questions: name of the depicted entity, visual complexity, and AoA), which led to a total of 156.620 responses across participants. Data from 1 participant were discarded from the analysis because he/she did not provide any answers. Therefore, data from a total of 329 participants were analyzed. For the name agreement measures, $D K$ responses represented only $0.76 \%$ of the data. Words with unambiguous spelling errors were recoded in their orthographically correct form. Alternative spellings were considered valid only when accepted by an online Greek dictionary. Considering that linguistic stimuli in experiments are usually used in their singular forms, picture names provided in plural were grouped with their singular form, with the exception of cases in which only the plural form was found across participants' answers (e.g., $\mu \alpha \lambda \lambda i \alpha$, meaning "hair").

The preferred answers and mean ratings corresponding to each picture are presented in a downloadable Appen- dix A (see the Supplemental Materials section). Starting from the leftmost column, the information provided for each item is (1) the item number with the most frequent Greek name and the most frequent English name, as reported in the Snodgrass and Vanderwart (1980) study; (2) the semantic category corresponding to each picture according to the category subdivisions reported by Rossion and Pourtois (2004) and by Snodgrass and Vanderwart; ${ }^{3}$ (3) the word length (measured as number of letters); and (4) the word frequency (measured as the number of occurrences per million words) of the dominant picture name taken from the GreekLex database (Ktori et al., 2008). In cases where the preferred picture name corresponded to a plural word form, the frequency values were those corresponding to the singular word form (e.g., for the name $\chi \varepsilon^{\prime} \lambda \eta$, meaning "lips," the frequency of $\chi \varepsilon i \lambda o \varsigma$ [lip] is given), since the database does not include plurals. It should be noted that word frequencies were not available in the database for four picture names, and also that word frequency for the picture of a rocking chair $\left(\kappa \alpha \rho \varepsilon \varepsilon^{\prime} \kappa \lambda \alpha\right.$ кovv $\iota \tau \tau \dot{\eta}$ ) could not be obtained, since the picture name was composed of two words. For the name agreement ratings (5), the information provided for each item is the total number of valid responses, the percentage of participants giving the most common name (\%), and the $H$ statistic, reflecting the percentage-agreement score taking into account the number of different names provided for a picture. For the visual complexity ratings (6) and for the AoA ratings (7), the information presented for each picture refers to the mean scores as provided according to the 1-to-5 Likert scales, along with their corresponding standard deviations (see also Table 1 for summary statistics of the measured variables).

The mean percentage of name agreement for the whole set of pictures was $87.45 \%$ across participants, showing that the majority of items were named with the same word by the vast majority of participants. In further detail, results regarding name agreement showed that 54 of 260 pictures were univocally named by all participants with the same word ( $H=0,100 \%$ agreement). Furthermore, 148 of the 260 pictures yielded name agreement percentages ranging from $80 \%$ to $99 \%, 29$ had percentages from $60 \%$ to $79 \%, 15$ from $50 \%$ to $59 \%$, and only 14 pictures had name agreement rating scores below $50 \%$. Even though percentages provide a straightforward measure of name agreement, the most informative and widely used measure of this variable is the $H$ statistic (e.g., Alario \&

Table 1

Summary Statistics for Name Agreement (Percentage of Participants Providing the Most Frequent Name and $H$ Values), Visual Complexity, and Age of Acquisition Ratings

\begin{tabular}{lccccc}
\hline & \multicolumn{2}{c}{$\begin{array}{c}\text { Name } \\
\text { Agreement }\end{array}$} & & $\begin{array}{c}\text { Visual } \\
\text { Complexity }\end{array}$ & $\begin{array}{c}\text { Age of } \\
\text { Acquisition }\end{array}$ \\
\cline { 2 - 3 } & $\%$ & $H$ & & 2.48 & 0.80 \\
$M$ & 87.45 & 0.55 & & 0.46 & 0.14 \\
$S D$ & 17.71 & 0.68 & & 2.00 & 2.00 \\
\hline
\end{tabular}

Note-Visual complexity and age of acquisition were rated on a 5-point Likert scale. 
Ferrand, 1999; Bonin et al., 2003; Rossion \& Pourtois, 2004; Snodgrass \& Vanderwart, 1980). The mathematical formula used to calculate the $H$ statistic was the same as in the original Snodgrass and Vanderwart study:

$$
H=\sum_{i=1}^{k} p_{i}\left[\log _{2}\left(1 / p_{i}\right)\right]
$$

where $k$ refers to the number of different names given to each picture and $p_{i}$ is the proportion of participants giving each name. Increasing $H$ values indicate decreasing levels of name agreement. When the same name for an item is given by all the participants, the obtained $H$ value is 0 , whereas, when two names are given with the exact same frequency, the item's $H$ value is 1 . The $H$ statistic is more informative regarding distribution than is the percentageagreement measure; for example, for two concepts with the same agreement percentage on the most frequent name, the $H$ values will differ depending on the number of alternative names given for each one of them.

A first set of correlation analyses was performed among the measured variables (see Table 2). In these analyses, we also included word frequency and word length, as taken from the GreekLex database. Results showed that the earlier a word was acquired (i.e., pictures eliciting lower AoA scores), the more participants agreed on its name (i.e., lower $H$ values), as shown by the significant correlation between these two variables $(r=.42, p<$ $.001)$. Contrarily, name agreement did not correlate significantly with word frequency $(r=-.07, p<.29)$. The correlation between AoA and word frequency, however, was significant $(r=-.30, p<.001)$, showing that higher frequency words are acquired earlier in life (see Alario \& Ferrand, 1999, for a similar pattern of results with a French-speaking sample). The strong correlation between AoA and word frequency has been observed in earlier picture naming research (e.g., Barry et al., 1997; Ellis \& Morrison, 1998), leading some authors to propose that frequency and AoA effects arise at the same stage of processing (e.g., Barry et al., 1997). However, the idea that AoA and frequency, although closely related, affect different processing stages has been recently put forward (e.g., Dent et al., 2008). Dent and colleagues have proposed that AoA is more closely related to object recognition and semantic processing stages, whereas word frequency is more closely linked to lexical-phonological retrieval stages. Consequently, it is feasible that in the process of providing a name for a given depicted object, without having to pronounce it (as in the case of the present study), the influence of AoA should be more noticeable than the influence of word frequency, coinciding with the pattern of correlations we obtained.

With regard to visual complexity, simpler drawings (i.e., pictures with lower visual complexity scores) corresponded to shorter names, as shown by the positive correlation between visual complexity and word length $(r=.22, p<.001)$. Also, participants rated pictures with higher frequency names as visually simpler, as indicated by the significant negative correlation between these two variables $(r=-.12, p<.05)$. It is thus not surprising
Table 2

Matrix of Correlations Among the Reported Measures

\begin{tabular}{lcccr}
\hline \multicolumn{1}{c}{ Measure } & NA $(H)$ & VC & AoA & Length \\
\hline Visual complexity & $.30^{* * *}$ & & & \\
Age of acquisition & $.42^{* *}$ & $.53^{* *}$ & & \\
Length & .03 & $.22^{* *}$ & $.23^{* *}$ & \\
Frequency & -.07 & $-.12^{*}$ & $-.30^{* *}$ & $-.17^{*}$ \\
\hline Note-NA $(H)$, name agreement; VC, visual complexity; AoA, age of
\end{tabular}
acquisition. ${ }^{*} p<.05 .{ }^{* *} p<.001$.

that the correlation between visual complexity and AoA was also significant $(r=.53, p<.001)$, with pictures depicting earlier acquired concepts (i.e., pictures with lower AoA values) being rated as less complex. Finally, the correlation between visual complexity and name agreement was also significant $(r=.30, p<.001)$, showing that simpler pictures produced higher name agreement scores (i.e., lower $H$ values).

A second set of correlation analyses was also performed in order to contrast specific values obtained in this normative study with those obtained in similar studies in other languages (for a similar procedure, see Alario \& Ferrand, 1999; Bonin et al., 2003; Dell'Acqua, Lotto, $\&$ Job, 2000). We compared the present data with (1) the data reported in the Rossion and Pourtois (2004) study for both the colorized and noncolorized versions of the S\&V picture set as rated by a group of French adults, (2) the data reported in the original Snodgrass and Vanderwart (1980) study with an American-English sample, (3) the data reported for the same line drawings by Alario and Ferrand (1999) with another French sample, and (4) the data reported by Sanfeliu and Fernandez (1996) with a Spanish sample (see Table 3). Our downloadable Appendix B contains name agreement [NA $(H)$ ], visual complexity (VC), and AoA values obtained for each item in the present study and in the parallel studies. These analyses showed that the name agreement $(H)$ values obtained in this study were highly correlated with the $H$ values reported in all the studies (Alario \& Ferrand, 1999, $r=$ $.38, p<.001$; Sanfeliu \& Fernandez, 1996, $r=.53, p<$ .001 ; Snodgrass \& Vanderwart, 1980, $r=.45, p<.001$ ), with the exception of the Rossion and Pourtois study. Considering that Rossion and Pourtois also used the colorized version of the S\&V pictures, one could have also expected a positive correlation between the present $H$ values and their $H$ values. However, it should be noted that with regard to name agreement consistency, there are

Table 3

Matrix of Correlations Between the Reported Measures for the Greek Sample and the American, French, and Spanish Samples for the Colorized and Noncolorized Versions of the Picture Set

\begin{tabular}{lccc}
\hline \multicolumn{1}{c}{ Sample } & NA $(H)$ & VC & AoA \\
\hline American (Snodgrass \& Vanderwart, 1980) & $.45^{* *}$ & $.82^{* *}$ & - \\
French colorized (Rossion \& Pourtois, 2004) & -.19 & $.84^{* *}$ & - \\
French noncolorized (Rossion \& Pourtois, 2004) & -.09 & $.86^{* *}$ & - \\
French (Alario \& Ferrand, 1999) & $.38^{* *}$ & $.80^{* *}$ & $.78^{* *}$ \\
Spanish (Sanfeliu \& Fernandez, 1996) & $.53^{* *}$ & $.68^{* *}$ & - \\
\hline
\end{tabular}

Note-NA $(H)$, name agreement; VC, visual complexity; AoA, age of acquisition. ${ }^{* *} p<.001$. 
not a priori reasons to expect significant cross-cultural correlations, given that the distribution of the alternative names for a given depicted object is not constant across languages (see Yoon et al., 2004). Thus, lower $H$ values for a specific picture in one language do not necessarily correspond to lower $H$ values for the same picture in a different language. As a paradigmatic example, consider the drawing of a lamp that in the Rossion and Pourtois study was uniquely named lampe by all participants $(H=0)$, whereas in the present study it yielded a more heterogeneous set of responses $(H=1.90 ; \pi \circ \rho \tau \alpha \tau i \phi, \phi \omega \tau \iota \sigma \tau \iota \kappa o ́$, $\lambda \alpha \mu \pi \alpha \tau \dot{\varepsilon} \rho, \lambda \alpha^{\prime} \mu \pi \alpha$, among others). Yet, it remains to be examined why the correlation of the name agreement values for the original black-and-white S\&V pictures obtained with the two French samples (Alario \& Ferrand, 1999, and Rossion \& Pourtois, 2004) was not significant $(r=-.49, p>.42)$.

One of the picture naming variables for which consistent values could be expected across languages is visual complexity, given that it is a purely perceptive measure. The results of the correlation analysis performed further confirmed this idea, since the visual complexity values obtained in the present study were highly correlated to those reported across all the parallel studies (Alario \& Ferrand, 1999, $r=.84, p<.001$; Rossion \& Pourtois, 2004, colorized, $r=.84, p<.001$, noncolorized, $r=.86$, $p<.001$; Sanfeliu \& Fernandez, 1996, $r=.68, p<.001$; Snodgrass \& Vanderwart, 1980, $r=.82, p<.001)$.

Another variable that could be expected to show crosslanguage significant positive correlations is AoA. However, of the above-mentioned studies, only a single one (Alario $\&$ Ferrand, 1999) provided AoA scores for the black-andwhite S\&V drawings. The correlation analysis between our study and the Alario and Ferrand study for AoA showed a significant positive correlation $(r=.78, p<.001)$.

In conclusion, the present study presents Greek normative data for the set of 260 color pictures from Rossion and Pourtois (2004). A large group of native Greek speakers (330 university students) provided name agreement responses for these pictures, as well as ratings corresponding to their visual complexity and age of acquisition. To our knowledge, the set of norms provided here constitutes the first normative study for a set of pictures in Modern Greek. We believe that these norms are a valuable tool for researchers throughout the different areas of cognitive psychology. Furthermore, we consider that these norms largely contribute to the recently initiated effort of standardizing the Greek language, as well as to enhancing the limited research on Greek.

\section{AUTHOR NOTE}

This research has been supported by Grants SEJ2006-09238/PSIC and CONSOLIDER-INGENIO 2010 CSD2008-00048 from the Spanish government and by Grant BFI05.310 from the Basque government. The authors thank Joana Cholin for her helpful comments on a draft of the manuscript, as well as all the students from the Department of Informatics of the National and Kapodistrian University of Athens for their participation. The full set of norms reported in the present study may be downloaded from the Web site of the Basque Center on Cognition, Brain and Language (www.bcbl.eu). Correspondence concerning this article should be addressed to M. Dimitropoulou, Departamento de Psicología Cognitiva, Universidad de La Laguna, 38205-Tenerife, Spain (e-mail: mdimitro@ull.es).

\section{REFERENCES}

Alario, F.-X., \& Ferrand, L. (1999). A set of 400 pictures standardized for French: Norms for name agreement, image agreement, familiarity, visual complexity, image variability, and age of acquisition. Behavior Research Methods, Instruments, \& Computers, 31, 531-552.

alario, F.-X., Ferrand, L., Laganaro, M., New, B., Frauenfelder, U. H., \& SEguI, J. (2004). Predictors of picture naming speed. Behavior Research Methods, Instruments, \& Computers, 36, 140-155.

Altmann, G. T. M., \& Kamide, Y. (2007). The real-time mediation of visual attention by language and world knowledge: Linking anticipatory (and other) eye movements to linguistic processing. Journal of Memory \& Language, 57, 502-518.

Barry, C., Morrison, C. M., \& Ellis, A. W. (1997). Naming the Snodgrass and Vanderwart pictures: Effects of age of acquisition, frequency and name agreement. Quarterly Journal of Experimental Psychology, 50A, 560-585.

Belke, E., Brysbaert, M., Meyer, A. S., \& Ghyselinck, M. (2005). Age of acquisition effects in picture naming: Evidence for a lexicalsemantic competition hypothesis. Cognition, 96, B45-B54.

Berman, S., Friedman, D., Hamberger, M., \& Snodgrass, J. G. (1989). Developmental picture norms: Relationships between name agreement, familiarity, and visual complexity for child and adult ratings of two sets of line drawings. Behavior Research Methods, Instruments, \& Computers, 21, 371-382.

Biederman, I. (1987). Recognition-by-components. Psychological Review, 94, 115-147.

Bogka, N., Masterson, J., Druks, J., Fragkioudaki, M., ChatZIPROKOPIOU, E.-S., \& ECONOMOU, K. (2003). Object and action picture naming in English and Greek. European Journal of Cognitive Psychology, 15, 371-403.

Bonin, P., Chalard, M., Méot, A., \& Fayol, M. (2002). The determinants of spoken and written picture naming latencies. British Journal of Psychology, 93, 89-144.

Bonin, P., Peereman, R., Malardier, N., Méot, A., \& Chalard, M. (2003). A new set of 299 pictures for psycholinguistic studies: French norms for name agreement, image agreement, conceptual familiarity, visual complexity, image variability, age of acquisition, and naming latencies. Behavior Research Methods, Instruments, \& Computers, 35, 158-167.

Cortese, M. J., \& Khanna, M. M. (2007). Age of acquisition predicts naming and lexical-decision performance above and beyond 22 other predictor variables: An analysis of 2,342 words. Quarterly Journal of Experimental Psychology, 60, 1072-1082.

Cuetos, F., Ellis, A. W., \& Alvarez, B. (1999). Naming times for the Snodgrass and Vanderwart pictures in Spanish. Behavior Research Methods, Instruments, \& Computers, 31, 650-658.

Cycowicz, Y. M., Friedman, D., Rothstein, M., \& Snodgrass, J. G. (1997). Picture naming by young children: Norms for name agreement, familiarity, and visual complexity. Journal of Experimental Child Psychology, 65, 171-237.

Damian, M. F., \& Martin, R. C. (1998). Is visual lexical access based on phonological codes? Evidence from a picture-word interference task. Psychonomic Bulletin \& Review, 5, 91-95.

Dell'Acqua, R., Job, R., \& Grainger, J. (2001). Is global shape sufficient for automatic object identification? Visual Cognition, 8 , 801-821.

Dell'Acqua, R., Lotto, L., \& Job, R. (2000). Naming times and standardized norms for the Italian PD/DPSS set of 266 pictures: Direct comparisons with American, English, French, and Spanish published databases. Behavior Research Methods, Instruments, \& Computers, 32, 588-615.

Dent, K., Johnston, R. A., \& Humphreys, G. W. (2008). Age of acquisition and word frequency effects in picture naming: A dual-task investigation. Journal of Experimental Psychology: Learning, Memory, \& Cognition, 34, 282-301.

Duñabeitia, J. A., Avilés, A., Afonso, O., Scheepers, C., \& CarREIRAS, M. (2009). Qualitative differences in the representation of 
abstract versus concrete words: Evidence from the visual-world paradigm. Cognition, 110, 284-292.

ElLIS, A. W., \& MorRISON, C. M. (1998). Real age-of-acquisition effects in lexical retrieval. Journal of Experimental Psychology: Learning, Memory, \& Cognition, 24, 515-523.

Hoshino, N., \& Kroll, J. F. (2008). Cognate effects in picture naming: Does cross-language activation survive a change of script? Cognition, 106, 501-511.

Huettig, F., \& Altmann, G. T. M. (2005). Word meaning and the control of eye fixation: Semantic competitor effects and the visual world paradigm. Cognition, 96, B23-B32.

Knupsky, A. C., \& Amrhern, P. C. (2007). Phonological facilitation through translation in a bilingual picture-naming task. Bilingualism Language \& Cognition, 10, 211-223.

KTORI, M., \& PITCHFORD, N. J. (2008). Effect of orthographic transparency on letter position encoding: A comparison of Greek and English monoscriptal and biscriptal readers. Language \& Cognitive Processes, 23, 258-281

Ktori, M., van Heuven, W. J. B., \& Pitchford, N. J. (2008). GreekLex: A lexical database of Modern Greek. Behavior Research Methods, $\mathbf{4 0}$ 773-783.

LACHMAN, R. (1973). Uncertainty effects on time to access the internal lexicon. Journal of Experimental Psychology, 99, 199-208.

Lachman, R., Shaffer, J. P., \& Hennrikus, D. (1974). Language and cognition: Effects of stimulus codability, name-word frequency, and age of acquisition on lexical reaction time. Journal of Verbal Learning \& Verbal Behavior, 13, 613-625.

Lloyd-Jones, T. J., \& NeTtLEMILL, M. (2007). Sources of error in picture naming under time pressure. Memory \& Cognition, 35, 816-836.

Martein, R. (1995). Norms for name and concept agreement, familiarity, visual complexity and image agreement on a set of 216 pictures. Psychologica Belgica, 35, 205-225.

MorRISON, C. M., \& ElLIS, A. W. (2000). Real age of acquisition effects in word naming and lexical decision. British Journal of Psychology, 91, $167-180$

Nishimoto, T., Miyawaki, K., Ueda, T., Une, Y., \& TaKahashi, M. (2005). Japanese normative set of 359 pictures. Behavior Research Methods, 37, 398-416.

Nisi, M., Longoni, A. M., \& Snodgrass, J. G. (2000). Misure italiane per l'accordo sul nome, familiarità ed età di acquisizione, per le 260 figure di Snodgrass e Vanderwart (1980) [Italian norms for name agreement, familiarity, and age of acquisition for the S\&V (1980) set of pictures]. Giornale Italiano di Psicologia, 27, 205-218.

Orfanidou, E., \& Sumner, P. (2005). Language switching and the effects of orthographic specificity and response repetition. Memory \& Cognition, 33, 355-369.

Paivio, A., Clark, J. M., Digdon, N., \& Bons, T. (1989). Referential processing: Reciprocity and correlates of naming and imaging. Memory \& Cognition, 17, 163-174.

PASHLER, H., \& Harris, C. R. (2001). Spontaneous allocation of visual attention: Dominant role of uniqueness. Psychonomic Bulletin \& Review, 8, 747-752.

Pind, J., JónsdótTIR, H., TRYggvadótTiR, H. B., \& Jónsson, F. (2000). Icelandic norms for the Snodgrass and Vanderwart (1980) pictures: Name and image agreement, familiarity, and age of acquisition. Scandinavian Journal of Psychology, 41, 41-48.

Price, C. J., \& Humphreys, G. W. (1989). The effects of surface detail on object categorization and naming. Quarterly Journal of Experimental Psychology, 41, 797-828.

Protopapas, A., Archonti, A., \& Skaloumbakas, C. (2007). Reading ability is negatively related to Stroop interference. Cognitive Psychology, 54, 251-282.

Rossion, B., \& Pourtois, G. (2004). Revisiting Snodgrass and Vanderwart's object pictorial set: The role of surface detail in basic-level object recognition. Perception, 33, 217-236.

Sanfeliu, M. C., \& Fernandez, A. (1996). A set of 254 Snodgrass-
Vanderwart pictures standardized for Spanish: Norms for name agreement, image agreement, familiarity, and visual complexity. Behavior Research Methods, Instruments, \& Computers, 28, 537-555.

Schriefers, H., JescheniaK, J. D., \& Hantsch, A. (2002). Determiner selection in noun phrase production. Journal of Experimental Psychology: Learning, Memory, \& Cognition, 28, 941-950.

Schriefers, H., Meyer, A. S., \& LeVelt, W. J. M. (1990). Exploring the time course of lexical access in production: Picture-word interference studies. Journal of Memory \& Language, 29, 86-102.

Shannon, C. E. (1949). The mathematical theory of communication. In C. E. Shannon \& W. Weaver (Eds.), The mathematical theory of communication (pp. 29-125). Urbana: University of Illinois Press.

SNODGRass, J. G., \& VANDERWART, M. (1980). A standardized set of 260 pictures: Norms for name agreement, image agreement, familiarity, and visual complexity. Journal of Experimental Psychology: Human Learning \& Memory, 6, 174-215.

SNODGRASs, J. G., \& YudiTSKY, T. (1996). Naming times for the Snodgrass and Vanderwart pictures. Behavior Research Methods, Instruments, \& Computers, 28, 516-536.

Suzuki, M., FujiI, T., Tsukiura, T., Okuda, J., Umetsu, A., NagaSAKA, T., ET AL. (2002). Neural basis of temporal context memory: A functional MRI study. NeuroImage, 17, 1790-1796.

VitKovitch, M., \& TYReLl, L. (1995). Sources of disagreement in object naming. Quarterly Journal of Experimental Psychology, 21A, 1155-1168.

Weekes, B. S., Shu, H., HaO, M., Liu, Y., \& Tan, L. H. (2007). Predictors of timed picture naming in Chinese. Behavior Research Methods, 39, 335-342.

Yoon, C., Feinberg, F., Luo, T., Hedden, T., Gutchess, A. H., Chen, H. Y., ET AL. (2004). A cross-culturally standardized set of pictures for younger and older adults: American and Chinese norms for name agreement, concept agreement, and familiarity. Behavior Research Methods, Instruments, \& Computers, 36, 639-649.

\section{NOTES}

1. During object recognition, texture and color information are considered to be of great relevance, especially for structurally similar objects and objects with a diagnostic color (e.g., fruits and vegetables). For instance, in the $\mathrm{S} \& \mathrm{~V}$ picture data bank, the image corresponding to "snow" could be very easily mistaken for a cloud. By including texture information, this confusion is resolved. Similarly, the addition of color is fundamental in some cases-for example, in discriminating between the images of an orange and a peach

2. In the present study, participants did not rate the pictures for image agreement (the degree to which images that participants generate to a picture's name agree with the picture's appearance). The omission of this variable was based on previous evidence suggesting that image agreement does not predict naming latencies when the drawings presented include color information (Rossion \& Pourtois, 2004; Weekes et al., 2007).

3. Note that the pictures were divided into 5 subsets according to Rossion and Pourtois's (2004) subdivision (animals, fruits/vegetables, body parts, man-made objects, and unclassified) and into 16 subsets based on Snodgrass and Vanderwart's (1980) subdivision (insects, musical instruments, vegetables, birds, carpenter's tools, clothing, fruits, animals, kitchen utensils, basic level, vehicles, human body parts, furniture weapons, building parts, and toys).

\section{SUPPLEMENTAL MATERIALS}

The name agreement, visual complexity, and age of acquisition norms reported in this and previous studies can be downloaded from brm .psychonomic-journals.org/content/supplemental.

(Manuscript received December 10, 2008; revision accepted for publication February 24, 2009.) 